Monatsschr Kinderheilkd 2022 · 170:204-206 https://doi.org/10.1007/s00112-022-01417-8 Angenommen: 3. Januar 2022

(c) The Author(s), under exclusive licence to Springer Medizin Verlag GmbH, ein Teil von Springer Nature 2022

\title{
Mehr \\ Arzneimitteltherapiesicherheit für Kinder
}

\author{
Wolfgang Rascher \\ Kinder- und Jugendklinik, Erlangen, Deutschland
}

Die Sicherheit von Arzneimitteln ist zum einen Aufgabe der pharmazeutischen Unternehmer und der Ordnungsgeber (z.B. Arzneimittelgesetz, europäische und deutsche Arzneimittelbehörden), damit ein wirksames und sicheres Produkt auf den Markt kommt und diese Sicherheit dauerhaft garantiert und überwacht wird (sicheres Produkt $\hat{=}$ Arzneimittelsicherheit). Zum anderen ist der Prozess der Verordnung, der Bereitstellung und der Gabe des Arzneimittels risikobehaftet; dieser gehört zu den Aufgaben der Ärzteschaft, die die Gabe in Verbindung mit den Apotheken steuert (sichere Gabe $\hat{=}$ Arzneimitteltherapiesicherheit). Pharmakovigilanz beschreibt die laufende und systematische Überwachung der Sicherheit eines Arzneimittels, mit dem Ziel, dessen unerwünschte Wirkungen zu entdecken, zu beurteilen und zu verstehen, um entsprechende Maßnahmen zur Minimierung des Risikos ergreifen zu können.

Pharmakotherapie ist bei Kindern viel aufwendiger und komplexer als bei Erwachsenen und damit ist auch das Risiko für unerwünschte Arzneimittelwirkungen (Nebenwirkungen) und Medikationsfehler höher. Dies liegt an der häufigen Anwendung von Medikamenten außerhalb der Zulassung (Off-label-Anwendung) mit fehlenden kindgerechten Darreichungsformen und an der gewichtsbezogenen Dosisberechnung. Trotzdem ist die Melderate eines Verdachts auf eine unerwünschte Arzneimittelwirkung bei Kindern geringer als bei Erwachsenen.

Eine Off-label-Anwendung ist bei Kindern nicht selten notwendig, um eine sachgerechte Pharmakotherapie zu gewährleisten. Nationale Nachschlagwerke (z.B. www.kinderformularium.de) liefern heute die Basis für eine evidenzbasierte Off-label-Anwendung [3]. Kinder- und Jugendärztinnen und -ärzte sollten heute wissen, wie die Evidenz für eine Off-label-Anwendung generiert wird, und wie sich nationale Arbeitsgruppen in einem europäischen Netzwerk zusammengetan haben, valide Informationen v.a. zu einer sicheren Offlabel-Anwendung zu liefern.

\section{》) Erhöhte Aufmerksamkeit redu- ziert vermeidbare Nebenwirkungen und Medikationsfehler}

Die Arzneimitteltherapiesicherheit bei Kindern wird durch Meldungen von Nebenwirkungen verbessert, wenn häufige oder schwerwiegende Nebenwirkungen ein Signal für ein hohes, vermeidbares Risiko liefern. Dies ist eindrucksvoll für die schon über Jahrzehnte verordneten Antihistaminika Dimenhydrinat und Diphenhydramin gezeigt worden, deren Zulassungen kürzlich für Säuglinge und Kleinkinder aus Sicherheitsbedenken eingeschränkt wurden [2]. Jede Meldung trägt dazu bei, die Information über ein Arzneimittel und damit die Sicherheit zu erhöhen.

Die zunehmende Digitalisierung in Krankenhäusern wird auch in Kinder- und Jugendkliniken vor einer elektronischen Arzneimittelverordnung nicht Halt machen. Dabei taucht die Frage auf, ob eine elektronische Verordnung, teilweise mit Verordnungsunterstützung, per se die Zahl der Medikationsfehler mit potenzieller und eingetretener Schädigung der Kinder vermindert. Wird die Literatur dazu analysiert, zeigt sich, dass diese Systeme einen gewissen Effekt haben, aber die Einführung einer guten Planung und ge- 
Hier steht eine Anzeige.

黑 Springer 
zielten Schulung aller Beteiligter bedarf; sonst können sie auch schaden.

Eltern behandeln nicht selten ihre Kinder selbst mit Medikamenten, die sie frei in der Apotheke kaufen können. In der repräsentativen Studie zur Gesundheit von Kindern und Jugendlichen in Deutschland (KiGGS) wurden Kindern nur 61,2\% der Medikamente verordnet, und $38,8 \%$ wurden in Form einer Selbstmedikation verabreicht, von denen etwa $30 \%$ außerhalb der Zulassung angewendet wurden [1]. Eltern müssen aber wissen, dass die Selbstmedikation auch Sicherheitsrisiken beinhaltet. Insbesondere bei Säuglingen und Kleinkindern ist Vorsicht geboten und der Rat der pädiatrischen Ärzteschaft hilfreich.

Erhöhte Aufmerksamkeit für die Arzneimitteltherapiesicherheit reduziert vermeidbare Nebenwirkungen und Medikationsfehler. Wenn es den Kinder- und Jugendärztinnen und -ärzten gelingt, vermeidbare Nebenwirkungen und Medikationsfehler zu reduzieren, wird die Zahl der arzneimittelbedingten stationären Aufnahmen um ein Drittel abnehmen (d.h. von $3 \%$ auf $2 \%$ aller stationären Aufnahmen). Dass dies möglich ist, will die Studie Verbesserung der Versorgung von Kindern und Jugendlichen mit Arzneimitteln durch Erhöhung der Arzneimitteltherapiesicherheit (KiDSafe, www. kidsafe.de) zeigen, deren Ergebnisse derzeit ausgewertet werden, und die zum Ziel hat, die Versorgung von Kindern und Jugendlichen mit Arzneimitteln durch die Erhöhung der Arzneimitteltherapiesicherheit zu verbessern.

Möge es gelingen, die Patientensicherheit bei Kindern mit mehr Aufmerksamkeit für eine sichere Arzneimittelverordnung und -gabe zu erhöhen.

\section{Korrespondenzadresse}

Prof. Dr. Dr. h.c. Wolfgang Rascher

Kinder- und Jugendklinik

Loschgestr. 15, 91054 Erlangen, Deutschland

wolfgang.rascher@uk-erlangen.de

Interessenkonflikt. W. Rascher hat Honorare für ein wissenschaftliches Gutachten vom Bundesinstitut für Arzneimittel und Medizinprodukte (BfArM) und für externe Sachverständigengutachten vom Institut für Qualität und Wirtschaftlichkeit im Gesundheitswesen (IQWiG) im Rahmen der frühen Nutzenbewertung erhalten.

\section{Literatur}

1. KnopfH, WolfIK, Sarganas G, Zhuang W, Rascher W, NeubertA (2013)Off-label medicine use in children and adolescents: results of a population-based study in Germany. Bmc Public Health 13:631

2. Moritz K, Rascher W, Toni I, Zahn J, Neubert A (2020) Zulassungseinschränkung der Antihistaminika der ersten Generation für Kinder unter 3 Jahren aufgrund eines negativen Nutzen-RisikoVerhältnisses - Wie Pharmakovigilanz gelingt. Monatsschr Kinderheilkd 168:143-147

3. Zahn J, Wimmer S, Rödle W, Toni I, Sedlmayr B, Prokosch HU, Rascher W, Neubert A (2021) Development and evaluation of a web-based paediatric drug information system for Germany. Pharmacy (Basel) 9(1):8

\section{Madeditorial}

\section{Hilfestellungen für den Editorial Manager}

Das Einreichungs- und Begutachtungssystem Ihrer Zeitschrift

Sowohl für die ganz alltäglichen Fragen in der Handhabung des Editorial Managers als auch für spezielle Problematiken finden Sie auf www.springermedizin.de/editorialmanager eine Vielzahl an Handreichungen, die Ihnen die Arbeit als Gutachter*in, Autor*in oder Herausgeber*in erleichtern.

Über Videos, einseitige Schritt-für-SchrittAnleitungen oder ein umfangreiches Manual werden Sie durch die einzelnen Punkte geführt, wie:

- Wie reiche ich ein Manuskript ein?

- Wie finde ich passende Gutachter*innen?

- Wie lade ich Gutachter*innen ein?

- Wie nehme ich ein Gutachten an bzw. lehne es ab?

- Wo erkenne ich, in welchem Status ein Mansukript ist?

- Wie ändere ich meine persönlichen Informationen?

- Wo kann ich meinen Urlaub eintragen?

\section{Zugang auch über QR-Code:}



